\title{
INSIGHTS
}

\section{Family reflections: prematurity}

\author{
Helen Ward ${ }^{1}$ \\ Pediatric Research (2019) 86:411-412; https://doi.org/10.1038/s41390-019-0339-2
}

Prematurity can be defined as birth before 37 weeks completed gestation. But for my family it is better defined as a constant leap into the unknown, our daughter forging her own path, in her own way, and in her own time. Born at 36 weeks and only a little over 3 lbs following intrauterine growth restriction, her early life was a somewhat overwhelming whirlwind of low blood sugars, inability to regulate temperature, feeding difficulties, jaundice, and episodes of apnoea, later to be joined by recurrent infections. Parenteral nutrition, incubators, phototherapy, nasogastric tubes and extensive blood tests and monitoring became her norm. This then gave way to frequent hospital visits and admissions for yet more infections, and then to delayed developmental milestones. Although now a healthy and developmentally 'on track' 3 year old, prematurity continues to impact our daughter. She has been placed in a different school year group to that which she should have been born into, has a petite stature, and a clutch of fears around medical staff and environments which she cannot explain, but with which we can increasingly draw links with her early experiences. For my husband and $\mathrm{I}$ it means living with the constant unknown; the dreaded possible longer term impacts which we were told may or may not come to fruition.

Through all of my daughter's early life, one fear overrode all others-was she in pain? If so, how could this be reduced, and how did I know that pain relief was working? There seemed so much uncertainty amongst the clinicians whom we trusted implicitly about this, and having since spoken to a multitude of parents and clinicians this seems a genuine unknown. At the time I tried to prepare for a range of outcomes, but the one that I could not reconcile with myself was that she had suffered. To this day certain smells and sounds, that after much reflection I realised link with the NICU, produce a strong fear response in my daughter, and although I have no way of knowing, I question why this is and fear that I know the answer. Research in this area would be welcomed by many parents I am sure, and I am heartened to hear that Bliss, the national charity for babies born premature and sick, are funding research in this area.

A further area where I would welcome research is post discharge care-both for the baby and their family. The intensity of the hospital experience was such that we monitored, recorded and considered in minute detail every aspect of her existence. On the day of discharge her feeds had to be $42 \mathrm{ml}$ exactly; a number that will forever be imprinted on my memory. Yet coming home we had little direction around many of the factors which had, up until then, been so closely controlled. We were instructed to follow her lead, and not to worry about numbers any more. Happily the implicit message was that our baby was now much better, and that we could go home to start the life with her that we had long awaited. But at the time it was terrifying. We had witnessed firsthand how quickly our baby could stop breathing and need intervention, and how wildly her blood sugar could drop, yet save for some leaflets from Bliss and a couple of short visits from outreach staff, we were now on our own. We were incredibly lucky, and with the support of family, our own internet research (again, thank you Bliss!), and a couple of panicked telephone calls to the NICU, we found our way through. Yet I wonder what the impact on parents more generally is at this time. Could research be carried out to determine how parental mental health can be better supported, and in doing so the health of the baby? Can more be done to identify how pre-discharge support can be optimised to better prepare parents to care for their baby at home? 'Rooming in' is a great opportunity for this, but in our experience it did little to teach us how to, for example, recognise the often very subtle signs of when she was becoming unwell, and when we needed to seek help. The extent to which we had to rely on information presented by charities such as Bliss surprised me, as did the length of time post discharge that we were referring back to this same information. Even now I find myself looking through their web pages as new questions arise.

Queries around consistency between the various levels of unit in which a baby is supported should also be addressed. We were incredibly lucky, in that our local hospital housed all three levels of care in the same building, and were staffed by the same team. As support was stepped down we continued to have contact with staff who had supported our daughter in her early days, and who knew what both she and us were capable of, for example giving tube feeds and undertaking her daily care. Yet speaking to other parents it seems that our experience is not always the case, and that a lot of babies need to be transferred between units. Not only do parents often have to travel long distances to be with their child, effective transfer of information regarding how parents had been involved in care, and what they had been trained to do/were allowed to do, seems to be limited. Parents I have spoken to talk about the confusion and frustration of having their involvement with their baby unnecessarily and abruptly reduced. Being involved in the care of my daughter was, I believe crucial to both her and us, and the way that we bonded with her. Any steps which can be taken towards finding ways to give greater consistency between units and to maximise parental involvement and family-centred care, will, again, be welcomed.

Finally, as we now enter the minefield of education, still prematurity is rearing its head. Should we delay the start of formal schooling? What kind of support does she need? I'm sure that these are the concerns of every parent, but I wonder if there should be more research to understand how to support school staff to understand the possible implications of prematurity, and to establish an effective monitoring mechanism to establish whether or not more support is required.

\footnotetext{
${ }^{1}$ Surrey, UK

Correspondence: Helen Ward (info@pedres.org)
}

Received: 6 February 2019 Accepted: 6 February 2019

Published online: 14 February 2019 
Without doubt, our little girl has benefitted hugely from research conducted into prematurity. We would not have her with us today had it not been for research. So to those engaged in such work I would like to say a huge thank you. Your time and efforts may sometimes feel fruitless and frustrating, but I promise you they are vitally important. Writing this I realise that whilst big, groundbreaking clinical trials are undoubtedly changing lives, so too are the smaller, perhaps underfunded projects which will change lives in more subtle ways. But they are all important to us as parents.
Having spoken to a number of parents of premature babies what strikes me is the level of trust which new parents have to have in medical staff, sometimes with no warning at all. For me that trust was underpinned by knowledge that clinicians were following evidence based practices, which are not possible without high quality research. As a parent on the NICU one clings to any glimmers of hope that can be found, and the more we know, the more we question and find out, the more there will be to cling to. So from the bottom of my heart, thank you for what you do. 